\title{
Predictores de síntomas de ansiedad, depresión y estrés a partir del brote epidémico de COVID-19
}

\author{
Mariela Andrades-Tobar ${ }^{1}$, Felipe E. García ${ }^{2,3}$, Pablo Concha-Ponce ${ }^{4}$, Carmen Valiente ${ }^{5}$ y \\ Cibelle Lucero ${ }^{1}$
}

\author{
${ }^{1}$ Escuela de Psicología y Terapia Ocupacional, Universidad Central, Chile. \\ ${ }^{2}$ Facultad de Ciencias Sociales y Comunicaciones, Universidad Santo Tomás, Chile. \\ ${ }^{3}$ Departamento de Psiquiatría y Salud Mental, Facultad de Medicina, Universidad de Concepción, Chile. \\ ${ }^{4}$ Facultad de Ciencias Sociales y Humanidades, Universidad Autónoma de Chile, Talca. \\ ${ }^{5}$ Facultad de Psicología. Departamento de Personalidad, Evaluación y Psicología Clínica, Universidad Complutense de Madrid, España.
}

Predictors of anxiety, depression, and stress symptoms from the COVID-19 outbreak

\begin{abstract}
The COVID-19 pandemic has caused an unprecedented global crisis, with dramatic changes in people's living conditions and mental health. The aim of this study is to establish predictors of depression, anxiety and stress in people affected by the epidemiological outbreak of COVID-19. Participants comprised 2,376 individuals over 18 years old, $79.6 \%$ women, who answered the Scale of Depression, Anxiety and Stress (DASS-21), in addition to scales to assess subjective severity, the search for social support and data related to COVID-19. It was observed that their main predictors were younger age, sleep problems, feeling lonely, not talking about their fears and subjective severity. The conclusion is that there are population groups at risk of experiencing higher levels of depression, anxiety and stress, which evidences the need to develop prevention and intervention strategies to reduce the psychological impact of the pandemic.
\end{abstract}

Keywords: Mental health; COVID-19; pandemic; confinement; quarantine.

Resumen: La pandemia de COVID-19 ha provocado una crisis mundial sin precedentes, con cambios drásticos en las condiciones de vida y en la salud mental de las personas. El propósito de este estudio es establecer predictores de depresión, ansiedad y estrés en personas afectadas por el brote epidemiológico de COVID-19. Participaron 2,376 personas mayores de 18 años, un 79.6\% mujeres, quienes contestaron la Escala de Depresión, Ansiedad y Estrés (DASS-21), además de escalas para evaluar la severidad subjetiva, la búsqueda de apoyo social y datos relacionados con COVID-19. Se observó que sus principales predictores fueron tener menor edad, problemas para dormir, sentirse solo, no hablar de sus temores y la severidad subjetiva. Se concluye que existen grupos de población en riesgo de experimentar niveles más altos de depresión, ansiedad y estrés, lo que evidencia la necesidad de desarrollar estrategias de prevención e intervención para reducir el impacto psicológico de la pandemia.

Palabras clave: Salud mental; COVID-19; pandemia; confinamiento; cuarentena.

\section{Introducción}

El 31 de diciembre de 2019, la Organización Mundial de la Salud (OMS, 2020) tomó conocimiento de una nueva

Recibido: 03 de septiembre 2020; aceptado:

Correspondencia: Dr. Felipe E. García, Barrio Universitario s/n, 4030000, Concepción, Chile. Correo-e: fgarciam@udec.cl neumonía viral de etiología desconocida, detectada en Wuhan, China. El 11 de marzo de 2020 la OMS declara la pandemia a nivel mundial por COVID-19. Este nuevo coronavirus, se ha extendido rápidamente por todo el mundo; al momento de redactar este artículo, el 20 de agosto de 2020, los contagiados ya suman más de 23 millones de personas en todo el mundo, y los fallecimientos alcanzan aproximadamente los 811.000 (Johns Hopkins University, 2020). 
En el continente americano los contagios siguen aumentando, con alrededor de 200.000 positivos diarios (OMS, 2020), convirtiéndose en el continente más afectado por la pandemia. En Chile, según el reporte del Ministerio de Salud del 24 de agosto de 2020, las cifras de casos confirmados de coronavirus ascienden a 399.568 y los fallecidos oficiales ya suman 10.916 en el país. Todo esto configura una situación sin precedentes.

Los brotes epidémicos, considerados como desastres naturales (Berman, 2009), impactan en forma intensa el comportamiento y el bienestar psicológico de gran parte de la población, provocando a menudo miedo y ansiedad (Balaratnasingam y Janca, 2006). Si bien los estudios disponibles sobre el efecto psicológico de enfermedades infeccionas similares al actual Covid-19 han sido escasos, los resultados muestran sistemáticamente que las personas afectadas muestran síntomas elevados de estrés, estrés postraumático y tendencias suicidas (Wheaton et al., 2012; Wu et al., 2009; Yip et al., 2010).

Respecto a la ansiedad, los estudios que se han realizado han arrojado diferentes resultados. Por ejemplo, Zhu et al. (2008) constataron que el 96.4\% del público chino encuestado mostró marcados cambios emocionales tras el brote de SARS en 2003. Sin embargo, usando el Inventario de Ansiedad Estado-Rasgo (State-Trait Anxiety Inventory), Cowling, et al. (2010) describieron que, durante la epidemia de gripe A de 2009 en Hong Kong, los encuestados tenían niveles bajos de ansiedad. Es muy probable que el impacto psicológico de una pandemia en la población esté condicionado, no solo por el tipo de instrumentos utilizados para medirlo, sino por las características de la infección, situación económica, circunstancias culturales y sociales de los países afectados.

Concretamente, en relación con la epidemia de COVID-19, los estudios que han utilizado instrumentos estandarizados, han arrojado datos similares. En un estudio realizado durante la fase inicial del brote en China, utilizando la escala GAD-7, han encontrado que el 20.4\% de las personas encuestadas en Shanghái presentaban niveles significativos de ansiedad, mientras que, en Wuhan, el epicentro de la crisis y en cuarentena durante el estudio de los autores, el 32.7\% mostraba ansiedad. Otros estudios recientes realizados fuera de Asia, utilizando las escalas validadas (GAD-7 y PHQ-9), también han encontrado altas tasas de ansiedad y depresión (Lee et al., 2020; Shevlin et al., 2020). En España, durante los meses de marzo y abril del 2020, se realizó también un estudio que estableció niveles elevados de problemas emocionales como depresión, ansiedad y estrés en una muestra amplia de personas expuestas al riesgo de contraer el virus (Sandín et al., 2020).
Un estudio realizado en China, a inicios de la pandemia, en la que se administró la Escala de Depresión, Ansiedad y Estrés (DASS-21), dio cuenta que el 16.5\% de los participantes mostró síntomas depresivos de moderados a graves; el 28.8\% síntomas de ansiedad de moderados a graves; y el $8.1 \%$ informó de niveles de estrés de moderados a graves (Wang et al., 2020). Por otra parte, un estudio en personas altamente susceptibles a la infección por coronavirus (trabajadores médicos), arrojó una tasa de prevalencia de estrés traumático de un $73.4 \%$, la depresión fue de $50.7 \%$, la ansiedad generalizada fue de $44.7 \%$, y el insomnio fue de $36.1 \%$ (Liu et al., 2020).

Entre los factores que inciden en una mayor vulnerabilidad a desarrollar problemas de salud metal ante situaciones de desastre, se encuentran el ser mujer (John et al., 2007; Xu y Song, 2011), la severidad objetiva del suceso, por ejemplo, haber sufrido la pérdida de alguien cercano, estar sin comida o agua (Irmansyah et al., 2010; Wickrama y Kaspar, 2007) y la severidad subjetiva, que refiere al riesgo percibido, miedo a morir, falta de control percibido, interpretación negativa de los recuerdos del evento y evaluación negativa de la respuesta de estrés (Lommen et al., 2009; Xu y Song, 2011).

Debido a que esta situación mundial es nueva y se encuentra en plena expansión, es aún prematuro estimar las consecuencias psicológicas y emocionales del brote epidémico de COVID-19. Sin embargo, las investigaciones realizadas en China y España apuntan a que el miedo a lo desconocido y la incertidumbre pueden llevar a evolucionar a distintos problemas de salud metal (Sandín et al. 2020; Shigemura et al., 2020).

El brote epidémico ha hecho que muchos países pidan a quienes han estado expuestos al riesgo de infección que se aíslen en sus casas o en una instalación de cuarentena especializada (Brooks et al., 2020). La aplicación de medidas de cuarentena, aislamiento forzado y distanciamiento social ha provocado cambios en la vida de millones de personas en el mundo. Estas son nuevas formas de experimentar la realidad que afectan la salud mental de la población, en especial a los grupos más vulnerables, como son las personas diagnosticadas con COVID-19 y el personal de salud (Maher et al., 2020), provocando síntomas de estrés, ansiedad, depresión, miedo, soledad y aumento en el consumo de alcohol y otras sustancias (Qiu et al., 2020; Shigemura et al., 2020).

Por lo tanto, en una emergencia de salud pública internacional como la actual, es relevante investigar el estado en que se encuentra la salud mental de la población durante este período de gran estrés representado por el riesgo de contagio por COVID-19 e identificar las principales necesidades de atención de salud mental de las personas afectadas, para desarrollar estrategias que permitan redu- 
cir el impacto causado por la pandemia. Considerando lo anteriormente señalado, el objetivo del presente estudio es analizar los niveles de depresión, ansiedad y estrés, y establecer sus predictores en personas expuestas al riesgo de contagio por la pandemia por Covid-19. Se espera encontrar un modelo satisfactorio que permita predecir los problemas de depresión, ansiedad y estrés que presente la población, que incluya variables socio-demográficas, relacionadas a la pandemia y psicológicas como la severidad subjetiva del evento y la búsqueda de apoyo social.

\section{Método}

\section{Participantes}

Participaron 2.376 personas, de las cuales 1.891 eran mujeres $(79.6 \%)$ y 485 varones $(20.4 \%)(M=34.25$ años; $D T=12.64$; rango 18 a 82 años). Un $84.7 \%$ de los participantes era de población chilena, un $11.7 \%$ eran mexicanos y un 3.6\% de otros países hispano-hablantes. Los participantes no recibieron ningún incentivo por contestar los cuestionarios.

\section{Diseño de investigación y procedimiento}

El presente estudio utilizó un diseño de investigación descriptivo y correlacional. Los datos fueron tomados en un solo corte temporal, por lo que se trata de un estudio transversal. La recolección de los datos se realizó online a través de un cuestionario auto administrado construido con Google Forms (https://www.google. com/forms/about) el que fue difundido por los investigadores del estudio, mediante correos electrónicos, plataformas virtuales y redes sociales. Los datos fueron recolectados desde el 27 de abril al 03 de mayo de 2020. El período de tiempo correspondió al primer brote en Chile de COVID-19 y varios confinamientos totales de la población. Se buscó reclutar a público en general, mayores de 18 años.

Antes de responder el set de cuestionarios, los participantes tuvieron acceso una carta de consentimiento en la que se informó del carácter voluntario de la participación y de la confidencialidad de los datos entregados, de acuerdo con las normas éticas vigentes para este tipo de estudios y según los cánones establecidos por la ley $\mathrm{N}^{\circ} 19.628$ de protección de datos de carácter personal en Chile. Luego de aceptar seguir participando, pudieron acceder a los cuestionarios propiamente tal. La recopilación de datos se llevó a cabo desde el 27 de abril al 03 de mayo de 2020. Este estudio fue aprobado por el Comité de Ética de la Investigación Científica de la Universidad Central de Chile ( ${ }^{\circ}$ 61/2020).

\section{Instrumentos}

Depression Anxiety Stress Scales (DASS-21; Lovibond y Lovibond, 1995). En Chile, este instrumento fue adaptado idiomática y culturalmente por Antúnez y Vinet (2012). Se trata de una escala de autoinforme, compuesta por 21 ítems, constituida por tres factores: Depresión, Ansiedad y Estrés, cada uno con siete ítems. Para responder, se presentan cuatro alternativas de respuesta en formato tipo Likert, las cuales van desde 0 («No describe nada de lo que me pasó o sentí en la semana») hasta 3 («Sí, esto me pasó mucho, o casi siempre»). La escala ha mostrado validez concurrente, con altas correlaciones con otras escalas (Mitchell et al., 2008) y un ajuste aceptable a un modelo de tres factores en muestras de habla hispana (Román, 2010). En Chile, este instrumento se ha adaptado para adolescentes y jóvenes (Antúnez y Vinet, 2012; Román et al., 2016), demostrando una confiabilidad satisfactoria, con valores $\alpha$ de Cronbach de .87 a .88 para la escala de Depresión, entre .72 a .79 para la escala de Ansiedad y de .82 a .83 para la escala de Estrés.

Escala de Severidad Subjetiva (ESS; Alzugaray et al., 2015). La escala consta de tres ítems que evalúan si la persona percibe que el evento alteró su vida, si lo siente como traumático y si piensa que es grave lo que le ha pasado. Cada una de ellas, es respondida en una escala Likert en un rango que va de 0 a 4 puntos. Su puntaje máximo es de 12 puntos. En el estudio de Alzugaray et al. (2015) mostró una consistencia interna adecuada $(\alpha=.86)$.

Brief COPE (Carver, 1997). Se utilizó la versión validada para población chilena de García et al., 2018). Se utilizaron las subescalas de búsqueda de apoyo social instrumental y emocional. El instrumento consta de cuatro ítems que se responden en una escala tipo Likert de 4 puntos, entre 0 («nunca hago esto») a 3 («hago siempre esto»). La sumatoria de estas dos escalas para la obtención de un puntaje único de búsqueda de apoyo social se ha utilizado en varios estudios, como el realizado por García y Miranda (2018) en población chilena afectada por un accidente, en el que se obtuvo un $\alpha$ de .71 .

Cuestionario de datos demográficos y datos relacionados con la COVID-19. El cuestionario consistió en preguntas que abarcaron variables sociodemográficas, otras relacionadas con el evento estresante (pandemia) y problemas de salud mental. Incluyó información relativa a sexo, edad, tener hijos, presencia de dificultades para dormir, sensación de soledad, hablar de los temores, tener algún familiar contagiado de COVID-19, tener trabajo y modalidad de trabajo actual. 


\section{Análisis de datos}

Se realizó inicialmente un análisis descriptivo de las variables de estudio. Luego, se establecieron las correlaciones entre las variables a través de la correlación $r$ de Pearson. A continuación, se realizaron comparaciones de grupo de acuerdo a la información recopilada en el cuestionario socio-demográfico, utilizando para ello la prueba $t$ de Student para variables independientes. Finalmente, se realiza una Regresión Lineal Múltiple Jerárquica para ver la influencia de las distintas variables sobre la depresión, la ansiedad y el estrés. Se consideró en un primer paso las variables estables, en un segundo paso las variables relacionadas a la respuesta ante el COVID-19 y en el tercer paso las variables psicológicas. Para la realización de los análisis se utilizó el programa
Statistical Package for the Social Sciences (SPSS) versión 21.0 (IBM Corp, 2011).

\section{Resultados}

Se realiza un análisis descriptivo de las variables de estudio. Los estadísticos de asimetría y curtosis se encuentran dentro del rango \pm 2 recomendado para el uso de estadísticos paramétricos (Bollen y Long, 1994). La consistencia interna de los instrumentos también fue adecuada. La información puede observarse en la Tabla 1. Al observar las medias de los tres problemas de salud mental evaluados en este estudio: depresión, ansiedad y estrés, el que presenta una media más elevada es el estrés, mostrando una diferencia significativa con respecto a la depresión y la ansiedad $\left(F_{(2,1330)}=114.792 ; p<.001\right)$.

Tabla 1. Estadísticos descriptivos y consistencia interna de los instrumentos $(n=2376)$

\begin{tabular}{lccccccc}
\hline \multicolumn{1}{c}{ Variable } & Min & Max & $M$ & $D E$ & Asimetría & Curtosis & $\alpha$ \\
Severidad subjetiva & 0 & 12 & 7.73 & 2.25 & -0.37 & -0.06 & 0.63 \\
Búsqueda de apoyo social & 0 & 12 & 4.54 & 2.82 & 0.28 & -0.42 & 0.86 \\
DASS - Depresión & 0 & 21 & 6.52 & 5.17 & 0.80 & -0.15 & 0.90 \\
DASS - Ansiedad & 0 & 21 & 4.98 & 4.63 & 1.09 & 0.61 & 0.87 \\
DASS - Estrés & 0 & 21 & 8.96 & 4.85 & 0.24 & -0.63 & 0.87 \\
\hline
\end{tabular}

Nota. DASS: Escala de depresión, ansiedad y estrés

Considerando los puntajes de corte propuestos por Román et al. (2016) para niveles leve o superior para cada uno de los problemas de salud mental, obtenidos en su estudio con población joven chilena (6 puntos o más para depresión y estrés y 5 puntos o más para ansiedad), el porcentaje de personas con depresión leve o superior es de $49.5 \%$, con ansiedad leve o superior es de un $43.3 \%$ y con estrés leve o superior es de un 74.1\%.El análisis de correlaciones muestra que la severidad subjetiva correlaciona en forma significativa con todas las variables, siendo la relación más alta con el estrés $(r=.41)$. La búsqueda de apoyo social correlaciona significativamente con todas las variables, excepto depresión, siendo las más altas con severidad subjetiva $(r=.17)$ y estrés $(r=$ $.10)$.

Tabla 2. Correlaciones $r$ de Pearson entre las variables $(n=2376)$

\begin{tabular}{lcccc}
\hline Variable & 2 & 3 & 4 & 5 \\
1. Severidad subjetiva & $0.17^{* * *}$ & $0.29^{* * *}$ & $0.33^{* * *}$ & $0.41^{* * *}$ \\
2. Búsqueda de apoyo social & na & -0.02 & $0.06^{* *}$ & $0.10^{* * *}$ \\
3. DASS - Depresión & & na & $0.71^{* * *}$ & $0.75^{* * *}$ \\
4. DASS - Ansiedad & & na & na \\
5. DASS - Estrés & & & \\
\hline
\end{tabular}

Nota. DASS: Escala de depresión, ansiedad y estrés.

${ }^{*} p<0.05 ; * * p<0.01 ; * * * p<0.001$

A continuación, se realizan análisis sobre la relación de algunas variables socio-demográficas o relacionadas con el evento estresante (pandemia) con problemas de salud mental. En primer lugar, la edad mostró una corre- 
lación significativa inversa con ansiedad $(r=-.26)$, estrés $(r=-.29)$ y depresión $(r=-.29)$. Al comparar por variables categóricas, se observa que ser mujer, no tener hijos, la presencia de dificultades para dormir, la sensación de soledad, no hablar de lo que les ocurre y no tener trabajo se relacionan con depresión, ansiedad y estrés. Por otro lado, tener un familiar contagiado con coronavi- rus no repercute en mayores problemas de salud mental respecto a quienes no tienen un familiar contagiado. Por último, teniendo trabajo, la modalidad de trabajo ejercida, ya sea presencial o teletrabajo, no genera diferencias en la salud mental. Los valores en las comparaciones se pueden observar en la tabla 3 .

Tabla 3. Comparaciones $t$ de Student en problemas de salud mental y sus factores de depresión, ansiedad y estrés de acuerdo a características socio-demográficas y del evento $(n=2376)$

\begin{tabular}{|c|c|c|c|c|c|c|}
\hline \multirow{2}{*}{ Variable } & \multicolumn{2}{|c|}{ DASS depresión } & \multicolumn{2}{|c|}{ DASS ansiedad } & \multicolumn{2}{|c|}{ DASS estrés } \\
\hline & $M(D E)$ & valor $t$ & $M(D E)$ & valor $t$ & $M(D E)$ & valor $t$ \\
\hline \multicolumn{7}{|l|}{ Sexo } \\
\hline Mujer & $6.77(5.17)$ & $4.610 * * *$ & $5.38(4.67)$ & $9.167 * * *$ & $9.38(4.77)$ & $8.360 * * *$ \\
\hline Hombre & $5.56(5.06)$ & & $3.40(4.14)$ & & $7.34(4.82)$ & \\
\hline \multicolumn{7}{|l|}{ ¿Tiene hijos? } \\
\hline No & $7.59(5.32)$ & $11.685 * * *$ & $5.58(4.76)$ & $7.170 * * *$ & $9.59(4.84)$ & $7.098 * * *$ \\
\hline Sí & $5.20(4.66)$ & & $4.24(4.36)$ & & $8.18(4.75)$ & \\
\hline \multicolumn{7}{|c|}{ ¿Algún familiar se ha contagiado de COVID-19? } \\
\hline No & $6.56(5.20)$ & 1.342 & $4.98(4.63)$ & 0.122 & $8.96(4.85)$ & -0.047 \\
\hline Sí & $6.02(4.74)$ & & $4.94(4.66)$ & & $8.98(4.88)$ & \\
\hline \multicolumn{7}{|c|}{ ¿Tiene dificultades para dormir? } \\
\hline No & $3.53(3.83)$ & $-20.730 * * *$ & $2.08(2.63)$ & $-26.263 * * *$ & $5.59(3.88)$ & $-25.235^{* * *}$ \\
\hline Sí & $7.66(5.19)$ & & $6.11(4.75)$ & & $10.28(4.54)$ & \\
\hline \multicolumn{7}{|c|}{ ¿Se ha sentido solo durante la pandemia? } \\
\hline No & $3.89(3.74)$ & $-26.344 * * *$ & $2.99(3.47)$ & $-21.374 * * *$ & $6.60(4.22)$ & $-24.339 * * *$ \\
\hline Sí & $8.72(5.18)$ & & $6.64(4.82)$ & & $10.94(4.44)$ & \\
\hline \multicolumn{7}{|c|}{ ¿Habla de sus temores sobre la pandemia con otros? } \\
\hline No & $8.31(5.83)$ & $9.536^{* * *}$ & $5.96(5.20)$ & $5.778 * * *$ & $9.87(5.31)$ & $5.247 * * *$ \\
\hline Sí & $5.86(4.73)$ & & $4.63(4.35)$ & & $8.63(4.62)$ & \\
\hline \multicolumn{7}{|c|}{ ¿Tiene trabajo actualmente? } \\
\hline No & $8.40(5.44)$ & $12.785^{* * *}$ & $6.21(5.00)$ & $9.253 * * *$ & $10.03(4.89)$ & $7.646^{* * *}$ \\
\hline Sí & $5.50(4.70)$ & & $4.29(4.28)$ & & $8.43(7.1)$ & \\
\hline \multicolumn{7}{|c|}{ ¿Cuál es su actual modalidad de trabajo? } \\
\hline Presencial & $5.48(4.86)$ & -0.148 & $4.53(4.43)$ & 1.109 & $8.21(4.99)$ & -0.971 \\
\hline Teletrabajo & $5.52(4.65)$ & & $4.23(4.21)$ & & $8.49(4.57)$ & \\
\hline
\end{tabular}

$* p<0.05 ; * * p<0.01 ; * * * p<0.001$

Se realiza una regresión lineal múltiple jerárquica con el fin de evaluar un modelo predictivo de los problemas de salud mental que las personas pueden desarrollar en relación al coronavirus. En el primer paso se incluyeron el sexo, la edad y si tiene o no hijos, por tratarse de variables estables, de modo de controlar su influencia. En el segundo paso se incluyeron las variables « ¿Tiene dificultades para dormir?», «¿Se ha sentido solo durante la pandemia?», «¿Habla de sus temores sobre la pandemia con otros?» y «¿Tiene trabajo actualmente?», es decir, las variables categóricas relacionadas a la pandemia. En el último paso se incluyeron el apoyo social y la severidad subjetiva, que son las variables psicológicas medidas por instrumentos validados. 
El modelo final para depresión resultó significativo, $F_{(9,1885)}=123.558, p<.001$, obteniendo un $R^{2}=.37$, lo que implica que el modelo predice el $37 \%$ de la varianza en la variable dependiente. El modelo final para la ansiedad también es significativo, $F_{(9,1885)}=123.558, p<$ .001 , con un $R^{2}$ de .34 . Lo mismo ocurrió con el modelo final para el estrés, $F_{(9,1885)}=149.357, p<.001$, con un $R^{2}$ de .42 . Los valores de los modelos pueden observarse en la Tabla 4.
Al observar los pesos de regresión, se constata que hay variables que influyen en todos los problemas de salud mental, como tener menor edad, problemas para dormir, sentirse solo, hablar de sus temores y la severidad subjetiva. Por otro lado, el sexo femenino predice más ansiedad y estrés pero no depresión. La presencia de hijos predice más estrés pero menos depresión. La falta de trabajo predice más depresión y más ansiedad, pero no predice el estrés. La búsqueda de apoyo social predice más estrés.

Tabla 4. Regresión lineal múltiple jerárquica para predecir problemas de salud mental $(n=2376)$

\begin{tabular}{|c|c|c|c|c|c|c|c|c|c|c|c|c|}
\hline \multirow{2}{*}{ Variable } & \multicolumn{4}{|c|}{ Depresión } & \multicolumn{4}{|c|}{ Ansiedad } & \multicolumn{4}{|c|}{ Estrés } \\
\hline & $R^{2}$ & $\Delta R^{2}$ & $\beta$ & Valor $t$ & $R^{2}$ & $\Delta R^{2}$ & $\beta$ & Valor $t$ & $R^{2}$ & $\Delta R^{2}$ & $\beta$ & Valor $t$ \\
\hline Paso 1 & $0.12 * * *$ & & & & $0.12^{* * *}$ & & & & $0.13^{* * *}$ & & & \\
\hline (Constante) & & & & $40.126^{* * *}$ & & & & $29.602 * * *$ & & & & $43.362 * * *$ \\
\hline Sexo $(0=$ mujer; $1=$ hombre $)$ & & & -0.11 & $-5.121 * * *$ & & & -0.18 & $-8.479^{* * *}$ & & & -0.18 & $-8.521^{* * *}$ \\
\hline Edad & & & -0.32 & $-9.939 * * *$ & & & -0.31 & $-10.866^{* * *}$ & & & -0.34 & $-12.323 * * *$ \\
\hline ¿Tiene hijos? $(0=$ no; 1 = sí) & & & -0.01 & $-1.970^{*}$ & & & 0.05 & 1.753 & & & 0.07 & $2.611^{* *}$ \\
\hline Paso 2 & 0.33 & $0.22 * * *$ & & & 0.28 & $0.17^{* * *}$ & & & 0.32 & $0.20 * * *$ & & \\
\hline (Constante) & & & & $15.470^{* * *}$ & & & & $11.707^{* * *}$ & & & & $20.521^{* * *}$ \\
\hline Sexo $(0=$ mujer; 1 = hombre $)$ & & & -0.04 & -1.835 & & & -0.10 & $-5.202^{* * *}$ & & & -0.09 & $-4.793^{* * *}$ \\
\hline Edad & & & -0.11 & $-4.124 * * *$ & & & -0.16 & $-6.099^{* * *}$ & & & -0.21 & $-8.163^{* * *}$ \\
\hline ¿Tiene hijos? (0 = no; 1 = sí) & & & -0.04 & -1.707 & & & 0.05 & 1.875 & & & 0.06 & $2.536^{* *}$ \\
\hline ¿Problemas para dormir? $(0=$ no; $1=$ sí $)$ & & & 0.21 & $10.351^{* * *}$ & & & 0.27 & $12.981^{* * *}$ & & & 0.29 & $14.272^{* * *}$ \\
\hline ¿Se ha sentido solo? $(0=$ no; 1 = sí $)$ & & & 0.33 & $16.195 * * *$ & & & 0.24 & $11.268^{* * *}$ & & & 0.29 & $14.129 * * *$ \\
\hline ¿Hablas de tus temores? $(0=$ no; $1=$ sí) & & & -0.12 & $-6.311 * * *$ & & & -0.05 & $-2.577^{* *}$ & & & -0.02 & -1.210 \\
\hline ¿Tienes trabajo? $(0=$ no; 1 = sí $)$ & & & -0.12 & $-5.911 * * *$ & & & -0.07 & $-3.289 * * *$ & & & -0.01 & -0.262 \\
\hline Paso 3 & 0.37 & $0.04 * * *$ & & & 0.34 & $0.06 * * *$ & & & 0.41 & $0.09 * * *$ & & \\
\hline (Constante) & & & & $8.570^{* * *}$ & & & & $3.522 * * *$ & & & & $9.579^{* * *}$ \\
\hline Sexo $(0=$ mujer; $1=$ hombre $)$ & & & -0.03 & -1.501 & & & -0.09 & $-4.774 * * *$ & & & -0.08 & $-4.220 * * *$ \\
\hline Edad & & & -0.15 & $-5.802 * * *$ & & & -0.21 & $-8.095 * * *$ & & & -0.27 & $-10.774^{* * *}$ \\
\hline ¿Tiene hijos? $(0=$ no; 1 = sí) & & & -0.05 & $-2.169^{*}$ & & & 0.04 & 1.489 & & & 0.05 & $2.166^{*}$ \\
\hline ¿Problemas para dormir $(0=$ no; $1=$ sí) & & & 0.17 & $8.604^{* * *}$ & & & 0.22 & $10.991^{* * *}$ & & & 0.23 & $12.107^{* * *}$ \\
\hline ¿Se ha sentido solo? $(0=$ no; 1 = sí) & & & 0.28 & $13.978 * * *$ & & & 0.18 & $8.731^{* * *}$ & & & 0.22 & $11.379^{* * *}$ \\
\hline ¿Hablas de tus temores? $(0=$ no; $1=$ sí $)$ & & & -0.13 & $-6.449^{* * *}$ & & & -0.08 & $-3.877^{* * *}$ & & & -0.07 & $-3.421^{* * *}$ \\
\hline ¿Tienes trabajo? $(0=$ no; 1 = sí) & & & -0.12 & $-6.079^{* * *}$ & & & -0.07 & $-3.516^{* * *}$ & & & -0.01 & -0.420 \\
\hline Severidad subjetiva & & & 0.20 & $10.273^{* * *}$ & & & 0.26 & $13.021^{* * *}$ & & & 0.31 & $16.390 * * *$ \\
\hline Búsqueda de apoyo social & & & -0.03 & -1.398 & & & 0.01 & 0.567 & & & 0.04 & $2.059^{*}$ \\
\hline
\end{tabular}

${ }^{*} p<0.05 ; * * p<0.01 ; * * * p<0.001$

\section{Discusión}

En la presente investigación se analizaron los niveles de estrés, ansiedad y depresión, la severidad subjetiva de lo vivi- do, la búsqueda de apoyo social, y variables sociodemográficas y de respuesta ante el brote epidémico del COVID-19.

Respecto a los problemas de salud mental, los puntajes de corte permitieron detectar la presencia de estados 
afectivos de depresión, ansiedad y estrés más altos que los encontrados en dos estudios sobre la pandemia en los que se administró el mismo instrumento, uno en China (Wang et al., 2020) y el otro en la Comunidad Autónoma Vasca (Ozamiz et al., 2020). Esto podría haber ocurrido, debido a que dichos estudios fueron realizados una vez iniciado el brote epidémico, y la presente investigación fue meses después, cuando la población ya estaba experimentando el confinamiento. Brooks et al. (2020) señalan que se espera un aumento de los problemas de salud mental según se vaya alargando el tiempo de confinamiento y aislamiento físico. Por otro lado, los puntajes de estos problemas de salud mental se asemejan a los encontrados en otro estudio con población china altamente susceptible a la infección por coronavirus (Liu et al., 2020).

Por otra parte, los niveles de estrés son superiores tanto en puntaje como en porcentaje de personas con nivel leve o superior, en comparación a depresión y ansiedad. Es posible que el principal problema de salud mental sea el estrés debido a la constante tensión que origina tanto la enfermedad y el riesgo de contagio, como los cambios en el estilo y la calidad vida de la población afectada, como el aislamiento social, la sobrecarga de información en los medios y el pánico de desabastecimiento (Wang et al., 2020). El porcentaje de personas con niveles leves o superiores al estrés es semejante al obtenido por Liu et al. (2020) para la sintomatología postraumática en población china.

Se observó que la severidad subjetiva referida a la pandemia influye en mayores problemas de salud mental. Esto se ha encontrado también en otros estudios que han señalado que la percepción de severidad de un evento estresante tendría una relación positiva con consecuencias negativas en la salud mental (Brewin et al., 2000; García et al., 2018; García y Miranda, 2019). Es probable que los mecanismos psicológicos que conducen a una consecuencia negativa en la salud mental se activen tras una percepción inicial sobre la gravedad del evento; por ejemplo, se ha encontrado que la severidad subjetiva activa procesos de rumiación intrusiva (Alzugaray et al., 2015; Cann et al., 2010).

Los resultados referentes al apoyo social, sugieren la existencia de una relación positiva con el estrés. Esto podría explicarse debido a que la tendencia a buscar la compañía de otras personas aumenta especialmente ante situaciones estresantes (Orcasita y Uribe, 2010). Esto es concordante con la ya clásica teoría de la comparación social de Festinger (1954), quien propuso que en situaciones de estrés surge la necesidad de buscar compañía de otras personas, pues de ese modo se obtiene información acerca de la situación estresante (lo que permitiría reducir la incertidumbre) y validar las propias reacciones (comparando sentimientos y conductas).

En cuanto a la edad, se encontraron medidas superiores en estrés, ansiedad y depresión en las personas de menor edad. Estos resultados concuerdan con una investigación realizada en España en paralelo al presente estudio (Sandín et al., 2020), en el cual las personas de menor edad (18 a 30 años) presentaron niveles superiores de malestar emocional en comparación a otros rangos de edad. Esto podría deberse, por una parte, a que, dada su etapa evolutiva, esta pandemia supone un quiebre y amenaza a los proyectos de los más jóvenes, como el comenzar o terminar con una carrera. Así también, en esta etapa de la vida, las relaciones sociales son vitales, el grupo de pares es muy relevante, y el confinamiento impide esos encuentros presenciales. Junto a esto, es importante señalar que la muestra más joven del presente estudio es mayoritariamente estudiante, por tanto, tuvieron que adaptarse a un nuevo contexto educativo sin clases presenciales. Los jóvenes, por lo demás, buscan información a través de medios formales en sus redes sociales, por lo que están más expuestos a información falsa y alarmante, afectando su salud mental (Bao et al., 2020). Por esto, la prevención y los programas de intervención para disminuir los niveles de problemas de salud mental creados y promovidos desde las mismas instituciones educativas serán fundamentales (Liu, et al., 2020).

Respecto al sexo, no se observaron diferencias entre hombres y mujeres respecto a la depresión. Sin embargo, ser mujer parece ser condición de riesgo para la ansiedad y estrés. Resultados semejantes fueron encontrados en el estudio de Sandín et al., (2020) en el que el perfil emocional asociado a COVID-19 en mujeres, sugiere un predominio de síntomas de estrés. Es posible, que, a partir de la pandemia, muchas mujeres tuvieron que trasladar sus rutinas laborales al hogar, desempeñando, muchas veces, una multiplicidad de roles. Estos resultados coinciden con algunas investigaciones que han encontrado que el desempeño de roles múltiples podría ser una fuente de estrés, pero a la vez, reportaría en general altos niveles de autoestima y sentido de eficacia y baja depresión (Gómez, 2001). Por tanto, sería relevante abordar respecto de cómo la sociedad debe adaptarse a esta nueva situación a través de una mirada de género.

Sentirse solo (a), también se relacionaría con mayores problemas de salud mental. La situación de confinamiento generó distancia física y en algunos casos, disminuyeron las relaciones sociales, lo que puede haber generado una sensación de soledad. Algunos estudios dan cuenta que la soledad tendría efectos adversos en el rendimiento cognitivo y se relacionaría con alteraciones 
del sueño, y efectos perjudiciales en la salud física, mental y en el bienestar social (Asher y Paquette, 2003; Wilson, et al., 2007).

Así mismo, no hablar de los temores y preocupaciones ante la pandemia, se relacionó con mayores problemas de salud mental. Según los estudios, compartir socialmente una emoción, hablar de lo sucedido, ayuda a amortiguar el impacto de los hechos traumáticos (Rimé et al., 2009). Por tanto, compartir las emociones y experiencias en relación con la pandemia, sería beneficioso para las personas afectadas, permitiendo el intercambio de experiencias con personas que están viviendo una experiencia similar y la adecuada expresión del malestar.

También se encontró que las dificultades para dormir se relacionan con mayores problemas de salud mental. Estos resultados coinciden con estudios realizados en China y España, que arrojaron que una mala calidad del sueño se asociaba a mayores problemas emocionales ( $\mathrm{Li}$ et al. 2020; Sandín et al., 2020). Ibáñez et al. (2020) incluso han identificado el insomnio como uno de los principales marcadores clínicos de problemas de salud mental ante la pandemia del coronavirus. En la situación actual de confinamiento, la mayoría de las personas están expuestas a situaciones estresantes sin precedentes y de duración desconocida. Esto puede interrumpir y alterar el sueño (Altena, et al., 2020), por lo que sería interesante analizar también la evolución de este proceso (Brooks, et al., 2020).

El presente estudio proporciona información relevante sobre las variables asociadas a problemas de salud mental en relación a la pandemia por COVID-19. Entre esta información encontramos las siguientes: 1) Los puntajes de corte permitieron detectar los altos niveles de depresión, ansiedad y estrés en población general durante la pandemia; 2) Las personas de menor edad presentaron niveles más altos de problemas de salud mental (depresión, ansiedad y estrés) que las personas de mayor edad; 3) Respecto al sexo, ser mujer parece ser condición de riesgo para la ansiedad y estrés; 4) Tener hijos predice mayores niveles de estrés; 5) La percepción subjetiva de severidad de la pandemia se asocia a mayores problemas de salud mental; 6) La soledad se relacionaría con mayores problemas de salud mental; 7) No hablar de los temores y preocupaciones ante la pandemia, se relacionó con mayores problemas de salud mental; 8) No tener trabajo predice mayores niveles de depresión y ansiedad; 9) Dificultades para dormir se relacionan con mayores problemas de salud mental y 10) La búsqueda de apoyo social se asocia a mayores niveles de estrés.

Este estudio presenta algunas limitaciones. La primera de ellas es que a pesar de que se contó con un buen número de participantes, el muestreo fue intencional por lo que no necesariamente los resultados son generalizables para toda la población. Por ejemplo, debido a las enormes dificultades que existen para aplicar encuestas presenciales, toda la población que se encuentra sin conexión regular a internet, la que probablemente representa a personas en condiciones de vida de mayor vulnerabilidad, han quedado excluidos del presente estudio. Una segunda limitación es que, dada su naturaleza transversal, no es posible concluir a partir de los resultados la existencia de relaciones causa-efecto entre las variables, por lo que la descripción de algunos hallazgos como los encontrados en la regresión, deben ser interpretados con cuidado. Una tercera limitación es la inexistencia de normas o puntajes de corte para la población encuestada en la escala DASS-21. Para el análisis mostrado se utilizaron normas chilenas para adolescentes y jóvenes, pero la muestra del estudio incluye a personas de otros países y de mayor edad, por lo que los porcentajes expuestos son tentativos. Una cuarta limitación es la ausencia de escalas validadas para medir variables psicológicas específicas asociadas a la pandemia, lo que obligó a usar ítems específicos y directos para evaluar algunas de ellas, por ejemplo, los problemas de sueño durante la pandemia, o la dificultad para compartir los temores y las preocupaciones sobre la pandemia con otras personas. El uso de ítems únicos impide la evaluación de algunas propiedades psicométricas como la consistencia interna.

Para futuros estudios, parece importante abordar algún tipo de consecuencia positiva tras lidiar con experiencias como una pandemia, lo que ya se ha encontrado en algunas investigaciones realizadas en España (Sandin et al., 2020; Vázquez et al., 2020) y que reflejan que ante situaciones adversas las personas también pueden desarrollar herramientas, percibir aprendizajes o favorecer experiencia personales positivas, algo que también merece ser reconocido y estudiado.

Los resultados de esta investigación significan un aporte respecto al conocimiento sobre el impacto de la pandemia en la salud mental de la población y permitirán desarrollar estrategias preventivas en población más susceptible, por ejemplo, a través de programas de apoyo para la salud mental en estudiantes que pueden proponer las mismas universidades, o desarrollar intervenciones clínicas que permitan hacer frente a las consecuencias emocionales de una pandemia que aún está en desarrollo. Relevante resulta también, perfilar estrategias de intervención clínica, orientadas a abordar el malestar emocional y el impacto psicológico causado por la pandemia por COVID-19.

\section{Conflictos de interés}

Los autores declaran no tener conflictos de intereses. 


\section{Referencias}

Altena, E., Baglioni, C., Espie, C. A., Ellis, J., Gavriloff, D., Holzinger, B., Schlarb, A., Frase, L., Jernelöv, S., \& Riemann, D. (2020). Dealing with sleep problems during home confinement due to the COVID-19 outbreak: Practical recommendations from a task force of the European CBT-I Academy. Journal of Sleep Research, e13052. https://doi. org/10.1111/jsr. 13052

Alzugaray, C., García, F., Reyes, A. y Álvarez, R. (2015). Propiedades psicométricas de una versión breve de la Escala de Rumiación Relacionada a un Evento en población chilena afectada por eventos altamente estresantes. Ajayu, 13(2), 183-198.

Antúnez, Z., y Vinet, E. V. (2013). Problemas de salud mental en estudiantes de una universidad regional chilena. Revista Médica de Chile, 141(2), 209-216.

Asher, S. R. \& Paquette, J. A. (2003) Loneliness and peer relations in childhood. Current Directions in Psychological Science, 12(3), 75-78. http://dx.doi.org/10.1111/1467-8721.01233

Balaratnasingam, S. \& Janca, A. (2006). Mass hysteria revisited. Current Opinion in Psychiatry, 19, 171-174. https://doi. org/10.1097/01.yco.0000214343.59872.7a

Berman, S. (2009). Pediatría en Desastres. Médica.

Bao, Y., Sun, Y., Meng, S., Shi, J., \& Lu, L. (2020). 2019-nCoV epidemic: Address mental health care to empower society. The Lancet, 395, 37-38. https://doi.org/10.1016/S0140-6736 (20)30309-3

Bollen, K. \& Long, J. S. (1994). Testing structural equation models. Sage.

Brewin, C. R., Andrews, B., \& Valentine, J. D. (2000). Metaanalysis of risk factors for posttraumatic stress disorder in trauma-expose dadults. Journal of Consulting and Clinical Psychology, 68(5), 748-766. https://doi.org/10.1037/0022006X.68.5.748

Brooks, S. K, Webster, R. K., Smith, L. E., Woodland, L., Wessely, S., Greenberg, N., \& Gideon, J. (2020). The psychological impact of quarantine and how to reduce it: Rapid review of the evidence. The Lancet, 395, 912-920. https://doi.org/10.1016/ S0140-6736(20)30460-8

Cann, A., Calhoun, L. G., Tedeschi, R. G., Triplett, K., Vishnevsky, T., \& Lindstrom, C. (2011). Assessing posttraumatic cognitive processes: The Event Related Rumination Inventory. Anxiety, Stress \& Coping, 24, 137-156. https://doi.org/10.1080/106158 06.2010 .529901

Carver, C. S. (1997). You want to measure coping but your protocol' too long: Consider the brief cope. International Journal of Behavioral Medicine, 4(1), 92-100. https://doi. org/10.1207/s15327558ijbm0401_6

Cowling, B. J., Ng, D.M.W., Ip, D.., Liao, Q., Lam, W., Wu, J., Lau, J., Griffiths, S. M., \& Fielding, R. (2010). Community psychological and behavioral responses through the first wave of the 2009 influenza A(H1N1) pandemic in Hong Kong. The Journal of Infectious Diseases, 6 (15) 867-876. https://doi. org/10.1086/655811

Festinger, L. (1954). A theory of social comparison processes. Human Relations, 7(2), 117-140. https://doi.org/10.1177/ 001872675400700202

García, F., Cova, F. y Reyes, A. (2014). Severidad del trauma, optimismo, crecimiento postraumático y bienestar en so- brevivientes de un desastre natural. Universitas Psychologica, 13(2), 15-24. https://doi.org/10.11144/Javeriana.UPSY13-2.stop

García, F. y Miranda, F. (2019). Predictores de sintomatología postraumática aguda y crecimiento post-estrés tras un accidente laboral reciente. Actualidades en Psicología, 33(126), 117-135. https://doi.org/10.15517/ap.v33i126.32680

García, F., Capponi, C., Hinrichs, F., Lillo, F., Rodríguez, C. y Sánchez, J. (2016). Violencia policial y afrontamiento: crecimiento postraumático en pobladores de una comunidad aislada del sur de Chile. Universitas Psychologica, 15(4), 1-12. http://dx.doi.org/10.11144/Javeriana.upsy15-4.vpac

García, F., Vega, N., Briones, F. y Bulnes, Y. (2018). Rumiación, crecimiento y sintomatología postraumática en personas que han vivido experiencias altamente estresantes. Avances en Psicología Latinoamericana, 36(3), 443-457. https://doi.org/ 10.12804/revistas.urosario.edu.co/apl/a.4983

Ibáñez, J. E., Alberdi, Í., \& Díaz, M. (2020). International mental health perspectives on the novel coronavirus SARS-CoV-2 pandemic. Revista de Psiquiatría y Salud Mental, 13(2): 111113. https://doi.org/10.1016/j.rpsm.2020.04.002

Irmansyah, I., Dharmono, S., Maramis, A., \& Minas, H. (2010). Determinants of psychological morbidity in survivors of the earthquake and tsunami in Aceh and Nias. International. Journal of Mental Health Systems 4(8). https://doi.org/10. 1186/1752-4458-4-8

John, P., Russell, S., \& Russell, P. (2007). The prevalence of posttraumatic stress disorder among children and adolescents affected by tsunami disaster in Tamil Nadu. Disaster Management \& Response 5(1),3-7. https://doi.org/10.1016/j.dmr.2006.11.001

Johns Hopkins University (2020). Coronavirus Resource Center. Disponible en: https://coronavirus.jhu.edu/

Lee, S. A., Jobe, M. C., \& Mathis, A. A. (2020). Mental health characteristics associated with dysfunctional coronavirus anxiety. Psychological Medicine, 1-2. https://doi.org/10.1017/ S003329172000121X

Liu, S., Yang, L., Zhang, C., Xiang, Y., Liu, Z., Hu, S., \& Zhang, B. (2020). Online mental health services in China during the COVID-19 outbreak. The Lancet Psychiatry, 7(4), e17-e18. https://doi.org/10.1016/S2215-0366(20) 30077-8

Lommen, M., Sanders, A., Buck, N., \& Arntz, A. (2009). Psychosocial predictors of chronic Post-Traumatic Stress Disorder in Sri Lankan tsunami survivors. Behaviour Research and Therapy, 47(1), 60-65. https://doi.org/10.1016/j.brat.2008. 10.009

Lovibond, S., \& Lovibond P. (1995). Manual for the Depression Anxiety Stress Scales. Psychology Foundation of Australia.

Maher, S., Hill, A. E., Britton, P., Fenichel, E. P., Daszak, P., Zambrana-Torrelio, C., \& Bayham, J. (2020). A COVID-19 risk assessment for the US Labor Force. medRxiv. https://doi. org/10.1101/2020.04.13.20063776

McAlonan, G., Lee, A., \& Cheung, V. (2007). Immediate and sustained psychological impact of an emerging infectious disease outbreak on health care workers. Canadian Journal of Psychiatry., 52, 241-247. http://doi: 10.1177/070674370705 200406.

Ministerio de Salud, Chile (2020). Informe epidemiológico $n .^{\circ} 37$. Enfermedad por SARS-CoV-2 (COVID-19). Minsal. Disponible en https://www.minsal.cl/37-informe-epidemiologicocovid-19/ 
Mitchell, M., Burns, N., \& Dorstyn, D. (2008). Screening for depression and anxiety in spinal cordin jury with DASS-21. Spinal Cord, 46, 547-551. https://doi.org/10.1038/sj. sc. 3102154

Orcasita, L. y Uribe, A. (2010). La importancia del apoyo social en el bienestar de los adolescentes. Psychologia: Avances de la Disciplina, 4(2),69-82.https://doi.org/10.21500/19002386.1151

Organización Mundial de la Salud. (2020). Brote de enfermedad por coronavirus (COVID-19). OMS. Disponible en: https:// www.who.int/es/emergencies/diseases/novelcoronavirus-2019

Ozamiz, N., Dosil., M., Picaza, M. y Idoiaga, N (2020). Niveles de estrés, ansiedad y depresión en la primera fase del brote del COVID-19 en una muestra recogida en el norte de España. Cadernos de Saúde Pública, 36(4), e00054020. https://doi. org/10.1590/0102-311x00054020.

Qiu, J., Shen, B., Zhao, M., Wang, Z., Xie, B., \& Xu, Y. (2020). A nation wide survey of psychological distress among Chinese people in the COVID-19 epidemic: Implications and policy recommendations. General Psychiatry, 33(2), e100213. https://doi.org/10.1136/gpsych-2020-100213

Rimé, B., Páez, D., Basabe, N., \& Martínez, F. (2009). Social sharing of emotion, post-traumatic growth, and emotional climate: Follow-up of Spanish citizen's response to the collective trauma of March 11th terrorist attacks in Madrid. European Journal of Social Psychology, 40, 1029-1045. https://doi.org/10.1002/ejsp.700

Román, M. (2010). Validación de la versión abreviada de las escalas de depresión, ansiedad y estrés (DASS - 21) en adolescentes estudiantes de enseñanza media de la comuna de Temuco. (Tesis de Magíster). Universidad de La Frontera, Temuco, Chile.

Román, F., Santibáñez, P. y Vinet, E. V. (2016). Uso de las Escalas de Depresión Ansiedad Estrés (DASS-21) como instrumento de tamizaje en jóvenes con problemas clínicos. Acta de Investigación Psicológica, 6(1), 2325-2336.

https://doi.org/10.1016/S2007-4719(16)30053-9

Román, F., Vinet, E. V. y Alarcón, A. M. (2014). Escalas de Depresión Ansiedad Estrés (DASS-21): Adaptación y propiedades psicométricas en estudiantes secundarios de Temuco. Revista Argentina Clínica Psicológica, 23(2), 179-190.

Sandín, B., Valiente, R., García-Escalera, J. y Chorot, R. (2020). Impacto psicológico de la pandemia de COVID-19: Efectos negativos y positivos en población española asociados al periodo de confinamiento nacional. Revista de Psicopatología y Psicología Clínica, 25(1), 1-22. https://doi.org/10.5944/ rppc. 27569

Shevlin, M., McBride, O., Murphy, J., Miller, J. G., Hartman, T. K., Levita, L., Mason, L., Martinez, A., McKay, R., Stocks, T., Bennett. K., Hyland, P., Karatzias, T., \& Bentall, R. (2020). Anxiety, depression, traumatic stress, and COVID-19 related anxiety in the UK general population during the COVID-19 pandemic. PsyArXiv. https://doi.org/10.31234/OSF.IO/HB6NQ
Shigemura, J., Ursano, R. J., Morganstein, J. C., Kurosawa, M., \& Benedek. D. M. (2020). Public responses to the novel 2019 coronavirus (2019-nCoV) in Japan: Mental health consequences and target populations. Psychiatry and Clinical Neurosciences, $\quad 74, \quad 281-282 . \quad$ https://doi.org/10.1111/ pcn. 12988

Vázquez, C., Valiente, C., García, F.E., Contreras, A., Peinado, V., Trucharte, A. \& Bentall, R. (2021). Post-traumatic growth and stress-related responses during the COVID-19 pandemic in a national representative sample: The role of positive core beliefs about the world and others. Journal of Happiness Studies. https://doi.org/10.1007/s10902-020-00352-3

Wang, C., Pan, R., Wan, X., Tan, Y., Xu, L., Ho, C.S., \& Ho, R.C. (2020). Immediate psychological responses and associated factors during the initial stage of the 2019 coronavirus disease (COVID-19) epidemic among the general population in China. International Journal of Environmental Research and Public Health, 17(5), 1-25. https://doi.org/10.3390/ ijerph17051729

Wheaton, M. G., Abramowitz, J. S., Berman, N. C., Fabricant, L. E., \& Olatunji, O. (2011). Psychological predictors of anxiety in response to the H1N1 (swine flu) pandemic. Cognitive Therapy and Research, 36(3), 210-218. https://doi. org/10.1007/s10608-011-9353-3

Wickrama, K. A. S. \& Kaspar, V. (2007). Family context of mental health risk in Tsunami-exposed adolescents: Findings from a pilot study in Sri Lanka. Social Science \& Medicine, 64(3), 713-723. https://doi.org/10.1016/j.socscimed.2006.09.031

Wilson, R. S., Krueger, K. R., Arnold, S. E., Schneider, J. A., Kelly, J. F., Barnes, L. L., ... \& Bennett, D. A. (2007). Loneliness and risk of Alzheimer disease. Archives of General Psychiatry, 64(2), 234-240. https://doi.org/10.1001/ archpsyc.64.2.234

Wu, P., Fang, Y., Guan, Z., Fan, B., Kong, J., Yao, Z., Liu, X., \& Hoven, C. W. (2009). The psychological impact of the SARS epidemic on hospital employees in China: Exposure, risk perception, and altruistic acceptance of risk. Canadian Journal of Psychiatry, 54, 302-311. https://doi. org/10.1177/070674370905400504

Xu, J. \& Song, X. (2011). Posttraumatic stress disorder among survivors of the Wenchuan earthquake 1 year after: Prevalence and risk factors. Comprehensive Psychiatry, 52(4), 431437. https://doi.org/10.1016/j.comppsych.2010.08.002

Yip, P. S. F., Cheung, Y. T., Chau, P. H., \& Law, Y. W. (2010). The impact of epidemic outbreak: The case of severe acute respiratory syndrome (SARS) and suicide among older adults in Hong Kong. Crisis: The Journal of Crisis Intervention and Suicide Prevention, 31(2), 86-92. https://doi. org/10.1027/0227-5910/a000015

Zhu, X., Wu, S., Miao, D., \& Li, Y. (2008). Changes in emotion of the Chinese public in regard to the SARS period. Social Behavior and Personality: An International Journal, 36(4) 447-453. https://doi.org/10.2224/sbp.2008.36.4.447 